\title{
Gain of a Smith-Purcell free-electron laser
}

\author{
H. L. Andrews and C. A. Brau \\ Department of Physics and Astronomy, Vanderbilt University, Nashville, Tennessee 37235, USA
}

(Received 13 April 2004; published 7 July 2004)

\begin{abstract}
A formula is derived for the small-signal gain of a Smith-Purcell free-electron laser. The theory describes the electron beam as a moving plasma dielectric, and assumes that the electron beam interacts with an evanescent mode traveling along the surface of a periodic waveguide with a rectangular profile. The phase velocity of the evanescent wave is synchronous with the electron velocity, but the group velocity is actually negative. The electron beam amplifies the evanescent wave, which does not itself radiate. According to this picture, the radiation observed emanating from the grating is Smith-Purcell radiation enhanced by the bunching of the electrons due to the interaction with the evanescent mode. There will also be radiation from the part of the evanescent mode that is outcoupled from the ends of the grating. This radiation appears at a lower frequency than the Smith-Purcell radiation. The new results explain both the gain and the radiation observed in the experiments of Urata and Walsh, and the cube-root current dependence of the gain inferred by Bakhtyari, Walsh, and Brownell.
\end{abstract}

DOI: 10.1103/PhysRevSTAB.7.070701

PACS numbers: 41.60.Cr, 07.57.Hm, 84.40.Fe

\section{INTRODUCTION}

There is currently substantial interest in the development of $\mathrm{THz}$ sources for applications to biophysics, medical imaging, nanostructures, and materials science [1]. At the present time, available $\mathrm{THz}$ sources fall into three categories: optically or electrically pumped gas lasers, solid state devices, and electron-beam driven devices. Gas lasers are commercially available and can provide hundreds of lines between 40 and $1000 \mu \mathrm{m}$, at powers ranging from $10 \mu \mathrm{W}$ to $1 \mathrm{~W} \mathrm{cw}$, and up to megawatts pulsed, but they are inherently not tunable. Solid state $\mathrm{THz}$ sources include $p$-type germanium lasers, quantum-cascade lasers, and excitation of numerous materials with subpicosecond optical laser pulses. Normally, $p$-type Ge lasers can be continuously tunable from 1 to $4 \mathrm{THz}$, but require a large $(1 \mathrm{~T})$ external magnetic field, must be operated at $20 \mathrm{~K}$, and have a limited repetition rate $(1 \mathrm{kHz})$ because of crystal heating [2]. Recently, a semiconductor heterostructure laser has produced up to $2 \mathrm{~mW}$ at $4.4 \mathrm{THz}$, at temperatures up to $50 \mathrm{~K} \mathrm{[3].} \mathrm{While} \mathrm{not}$ tunable, such lasers can be fabricated to produce the frequency desired. Subpicosecond electromagnetic pulses can be used as broad band sources of THz radiation. Short pulses can be created by optical rectification of subpicosecond infrared laser pulses [4] or by optically switching the photoconductor in a small diode antenna [5]. These broad band pulses are good for pump-probe or time-resolved experiments [6], but are less well suited to spectroscopy.

Electron-beam driven sources include backward-wave oscillators (BWOs), synchrotrons, and free-electron lasers (FELs). The shortest wavelength produced to date by a BWO was $0.25 \mathrm{~mm}$, in 1979 [7]. Current commercially available BWOs produce milliwatts from 30-1000 GHz. Modern synchrotrons with short electron bunches, such as BESSY II in Berlin [8], and recirculating linacs like the FEL at Jefferson Laboratory [9], produce many watts of broad band radiation out to about $1 \mathrm{THz}$. Conventional FELs have also been operated in the $\mathrm{THz}$ region. The millimeter-wave and far-infrared FELs at University of California Santa Barbara together operate between $2.5 \mathrm{~mm}$ and $338 \mu \mathrm{m}$ and produce $1-15 \mathrm{~kW}$ of power in microsecond pulses [10]. Coherently enhanced $\mathrm{THz}$ spontaneous emission from relativistic electrons in undulators has been recently observed at ENEA-Frascati with $\mathrm{kW}$ power levels in microsecond pulses [11]. However, all these sources (synchrotrons, undulators, and FELs) require large facilities.

An interesting opportunity for a convenient, tunable, narrow band source is presented by the recent development of a tabletop Smith-Purcell free-electron laser at Dartmouth [12]. This device has demonstrated superradiant emission in the spectral region from 300-900 $\mu \mathrm{m}$, but barely exceeded threshold. To improve on this performance, it will be necessary to develop electron beams with improved brightness [13] and a better understanding of how these devices operate.

Smith-Purcell radiation is emitted when an electron passes close to the surface of a grating, as indicated in Fig. 1. The virtual photons of the electron field are scattered by the grating, and the wavelength $\lambda$ of the radiation observed at the angle $\theta$ from the normal is

$$
\frac{\lambda}{L}=\frac{1}{\beta}-\sin \theta,
$$

where $\beta c$ is the electron velocity, $L$ the grating period, and $c$ the speed of light. This prediction has been amply confirmed by experiments [12]. The angular and spectral intensity of Smith-Purcell radiation is described by the theory of van den Berg and Tan [14-16].

When the current in the electron beam is sufficiently high, the electrons interact with the fields above the 


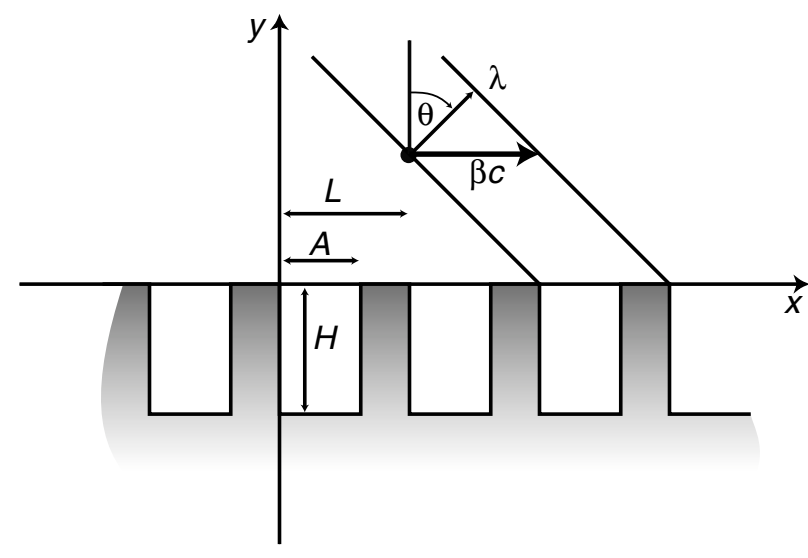

FIG. 1. Formation of Smith-Purcell radiation by an electron moving parallel to the surface of a grating with rectangular grooves.

grating. This causes nonlinear bunching of the electrons in the beam, which enhances the Smith-Purcell radiation. This was first observed experimentally by Urata et al. [12], although related radiation from traveling-wave tubes and similar microwave devices based on slowwave interactions in periodic structures had previously been observed [17]. Several theories have been proposed to describe the operation of a Smith-Purcell FEL [1820]. In particular, Schaechter and Ron proposed a theory based on the interaction of an electron-beam with a wave traveling along the grating [19]. The interaction is found to amplify waves incident on the grating and reflected by it, with a gain that depends on the reflection matrix of the grating. They find that the gain is proportional to the cube root of the electron-beam current, which agrees with the behavior of Cherenkov free-electron lasers [21] and other slow-wave devices [17]. More recently, Kim and Song have proposed a theory in which they assume that the electrons interact with a wave that travels along the surface of the grating [20]. They assume that at least one Fourier component of the traveling wave is radiative, and they find that the gain is proportional to the square root of the electron-beam current. The present theory is based on the interaction of the electron beam with a nonradiating, evanescent mode of the grating. The predicted gain is proportional to the cube root of the electron-beam current.

\section{THEORY}

The theory is straightforward. A uniform plasma traveling in the positive $x$ direction with velocity $\beta c$ is assumed to fill the region $y>0$ above the grating shown in Fig. 1. In the rest frame of the plasma, the magnetic susceptibility vanishes and the dielectric susceptibility is [22]

$$
\chi_{e}^{\prime}=-\frac{\omega_{p}^{\prime}}{\omega^{\prime 2}}
$$

where $\omega^{\prime}$ is the optical frequency and

$$
\omega_{p}^{\prime 2}=\frac{n_{e}^{\prime} q^{2}}{\varepsilon_{0} m}
$$

the plasma frequency in the plasma rest frame, in which $n_{e}^{\prime}$ is the electron density, $q$ the electron charge, $m$ the electron mass, and $\varepsilon_{0}$ the permittivity of free space (SI units are used throughout). For a wave of the form $\exp \left[i\left(\mathbf{k}^{\prime} \cdot \mathbf{r}^{\prime}-\omega^{\prime} t^{\prime}\right)\right]$, where $\mathbf{k}^{\prime}$ is the wave vector, $\mathbf{r}^{\prime}$ the position, and $t^{\prime}$ the time in the plasma rest frame, we find from the wave equation that

$$
\frac{\omega^{\prime 2}}{c^{2}}-\mathbf{k}^{\prime} \cdot \mathbf{k}^{\prime}=k^{\prime \alpha} k_{\alpha}^{\prime}=-\chi_{e}^{\prime} \frac{\omega^{\prime 2}}{c^{2}},
$$

where $k^{\prime \alpha}=\left(\omega^{\prime} / c, \mathbf{k}^{\prime}\right)$. But $k^{\prime \alpha} k_{\alpha}^{\prime}$ is a Lorentz invariant, so in the laboratory frame this becomes

$$
k^{\alpha} k_{\alpha}=\frac{\omega^{2}}{c^{2}}-\mathbf{k} \cdot \mathbf{k}=-\chi_{e}^{\prime} \frac{\omega^{\prime 2}}{c^{2}}=\frac{\omega_{p}^{2}}{\gamma c^{2}},
$$

where $\omega$ is the frequency and $\mathbf{k}$ the wave vector in the laboratory frame, $\gamma=1 / \sqrt{1-\beta^{2}}$, and the plasma frequency in the laboratory frame is $\omega_{p}^{2}=\gamma \omega_{p}^{\prime 2}$, due to Lorentz contraction.

The polarization in the laboratory frame is given by the relativistically correct constitutive relation [23]

$$
\mathbf{P}-\frac{\mathbf{v} \times \mathbf{M}}{c^{2}}=\varepsilon_{0} \chi_{e}^{\prime}(\mathbf{E}+\mathbf{v} \times \mathbf{B}),
$$

where $\mathbf{M}$ is the magnetization, $\mathbf{v}=\beta c \hat{\mathbf{x}}$ the velocity, $\mathbf{E}$ the electric field, and $\mathbf{B}$ the magnetic field. From the $x$ component of this equation we see that the displacement is

$$
D_{x}=\varepsilon_{0} E_{x}+P_{x}=\varepsilon_{0}\left(1+\chi_{e}^{\prime}\right) E_{x}
$$

The frequency in the plasma rest frame is

$$
\frac{\omega^{\prime}}{c}=\gamma\left(\frac{\omega}{c}-\beta k_{x}\right)
$$

so the dielectric susceptibility

$$
\chi_{e}^{\prime}=\frac{-\omega_{p}^{2}}{\gamma^{3}\left(\omega-\beta c k_{x}\right)^{2}}
$$

diverges at the synchronous point

$$
\omega=\beta c k_{x} .
$$

In the following we confine our attention to TM waves, for which the magnetic field in the $x$ direction vanishes. To describe the wave in the evanescent region, we take advantage of Floquet's theorem and expand the $\mathbf{E}$ and $\mathbf{H}$ fields in the form

$$
E_{x}=\sum_{p=-\infty}^{\infty} E_{p} e^{-\alpha_{p} y} e^{i p K x} e^{i(k x-\omega t)},
$$




$$
H_{z}=\sum_{p=-\infty}^{\infty} H_{p} e^{-\alpha_{p} y} e^{i p K x} e^{i(k x-\omega t)}
$$

where $E_{p}$ and $H_{p}$ are constants, and

$$
K=\frac{2 \pi}{L}
$$

is the grating wave number. For convenience, in the following we use $k$ to denote the $x$ component of the wave vector, rather than its magnitude. From the wave equation we find that

$$
\alpha_{p}^{2}=(k+p K)^{2}-\frac{\omega^{2}}{c^{2}}+\frac{\omega_{p}^{\prime 2}}{c^{2}} .
$$

Computations show that the wave is evanescent (nonradiative), since $\alpha_{p}^{2}>0$ for all $p$. To satisfy the boundary condition that the wave vanish in the limit $y \rightarrow \infty$, we chose the negative root $\alpha_{p}<0$. From the MaxwellAmpere law we find that

$$
\alpha_{p} H_{p}=i \varepsilon_{0} \omega\left(1+\chi_{p}^{\prime}\right) E_{p},
$$

where the dielectric susceptibility at the frequency of the $p$ th component is

$$
\chi_{p}^{\prime}=\frac{-\omega_{p}^{2}}{\gamma^{3}[\omega-\beta c(k+p K)]^{2}} .
$$

When the wave is nearly synchronous, the susceptibility is nearly divergent only for $p=0$, so we write (15) in the form

$$
H_{p}=i \varepsilon_{0} \frac{\omega}{\alpha_{p}}\left(1+\delta_{p 0} \chi_{0}^{\prime}\right) E_{p}
$$

In the grooves of the grating we expand the fields in the Fourier series

$$
\begin{aligned}
E_{x} & =\sum_{n=0}^{\infty} \bar{E}_{n} \cos \left(\frac{n \pi x}{A}\right) \frac{\sinh \left[\kappa_{n}(y+H)\right]}{\cosh \left[\kappa_{n} H\right]} e^{-i \omega t}, \\
H_{z} & =\sum_{n=0}^{\infty} \bar{H}_{n} \cos \left(\frac{n \pi x}{A}\right) \frac{\cosh \left[\kappa_{n}(y+H)\right]}{\sinh \left[\kappa_{n} H\right]} e^{-i \omega t},
\end{aligned}
$$

where $\bar{E}_{n}$ and $\bar{H}_{n}$ are constants, $A$ is the width of the groove, and $H$ the depth. These expressions satisfy the boundary conditions that $E_{x}$ vanish at the bottom of the groove $(y=-H)$, and $\partial H_{z} / \partial x$ vanish at the sides of the groove $(x=0, A)$. From the wave equation we find that

$$
\kappa_{n}^{2}=\left(\frac{n \pi}{A}\right)^{2}-\frac{\omega^{2}}{c^{2}}
$$

and from the Maxwell-Ampere law we get

$$
\bar{H}_{n}=-i \varepsilon_{0} \frac{\omega}{\kappa_{n}} \tanh \left(\kappa_{n} H\right) \bar{E}_{n} .
$$

Across the interface between the grating and the electron beam, the tangential component of the electric field is continuous. Since the tangential field vanishes on the surface of the conductor, we get [suppressing the $\exp (-i \omega t)$ dependence]

$$
\sum_{p=-\infty}^{\infty} E_{p} e^{i(k+p K) x}= \begin{cases}\sum_{n=0}^{\infty} \bar{E}_{n} \cos \left(\frac{n \pi x}{A}\right) \tanh \left(\kappa_{n} H\right) & \text { for } 0<x<A \\ 0 & \text { for } A<x<L .\end{cases}
$$

If we multiply by $\exp [-i(k+q K) x]$ and integrate over $0<x<L$, we get

$$
E_{q}=\sum_{n=0}^{\infty} \bar{E}_{n} \tanh \left(\kappa_{n} H\right) \frac{K_{q n}}{L},
$$

where

$$
K_{q n}=i A \frac{(k+q K) A}{(k+q K)^{2} A^{2}-n^{2} \pi^{2}}\left[(-1)^{n} e^{-i(k+q K) A}-1\right] .
$$

Likewise, the tangential component of the magnetic field must be continuous across the interface, so

$$
\sum_{p=-\infty}^{\infty} H_{p} e^{i(k+p K) x}=\sum_{n=0}^{\infty} \bar{H}_{n} \cos \left(\frac{n \pi x}{A}\right) \operatorname{coth}\left(\kappa_{n} H\right) .
$$

If we multiply by $\cos (m \pi x / A)$ and integrate over $0<x<$ $A$, we get

$$
\bar{H}_{m} \frac{1+\delta_{m 0}}{2} \operatorname{coth}\left(\kappa_{m} H\right)=\sum_{p=-\infty}^{\infty} H_{p} \frac{K_{p m}}{A} .
$$

If we substitute (17) and (21) into (26), substitute the sum (23) for $E_{p}$, and reverse the order of summation, we get

$$
\bar{E}_{m}=\sum_{n=0}^{\infty} C_{m n} \bar{E}_{n}
$$

where

$$
C_{m n}=i \frac{2 \kappa_{m} A}{m \pi} \frac{\tanh \left(\kappa_{n} H\right)}{1+\delta_{m 0}} \sum_{p=-\infty}^{\infty} \frac{k+p K}{\alpha_{p}}\left(1+\delta_{p 0} \chi_{0}\right) K_{p m} K_{p n} .
$$

For a solution of (27) to exist, the determinant of the coefficients must vanish,

$$
\left|C_{m n}-\delta_{m n}\right|=0 \text {. }
$$

This is the dispersion relation, and its roots give us the functional dependence $\omega(k)$. 
For convenience we write

$$
C_{m n}=R_{m n}+\chi_{0} S_{m n}
$$

where after some algebra we find that

$$
R_{m n}=\frac{\tanh \left(\kappa_{n} H\right)}{1+\delta_{m 0}} \sum_{p=-\infty}^{\infty} \frac{\kappa_{m} A}{\alpha_{p} L} \frac{4}{(k+p K)^{2} A^{2}-m^{2} \pi^{2}} \frac{(k+p K)^{2} A^{2}}{(k+p K)^{2} A^{2}-n^{2} \pi^{2}} \begin{cases}(-1)^{m} \cos [(k+p K) A]-1 & \text { for } m+n=\text { even } \\ i(-1)^{m} \sin [(k+p K) A] & \text { for } m+n=\text { odd },\end{cases}
$$

and

$$
S_{m n}=\frac{\tanh \left(\kappa_{n} H\right)}{1+\delta_{m 0}} \frac{\kappa_{m} A}{\alpha_{0} L} \frac{4}{k^{2} A^{2}-m^{2} \pi^{2}} \frac{k^{2} A^{2}}{k^{2} A^{2}-n^{2} \pi^{2}} \begin{cases}(-1)^{m} \cos [k A]-1 & \text { for } m+n=\text { even } \\ i(-1)^{m} \sin [k A] & \text { for } m+n=\text { odd }\end{cases}
$$

In the absence of the electron beam, the dispersion relation is

$$
\left|R_{m n}-\delta_{m n}\right|=0
$$

Some simple computations carried out using MATHCAD are shown in Figs. 2 and 3 for the parameters used in the experiments of Urata and Walsh, summarized in Table I. In the dispersion diagram, Fig. 2, the operating point of the laser is the point where the beam line, $\beta k$, intersects the dispersion curve. As shown there, for $40-\mathrm{keV}$ electrons the intersection occurs at a point $k / K>0.5$, where $d \omega / d k<0$. We see from these results that while the evanescent wave travels with a positive phase velocity equal to the electron velocity, the group velocity is negative, in the manner of a backward-wave oscillator. It is important to note that the waves are evanescent (they vanish exponentially above the grating) over the entire range explored by Urata and Walsh.

The computations also show that the dispersion relation is accurately described (within a few percent) by (33) even if just a single element (that is, $m=n=0$ ) is used

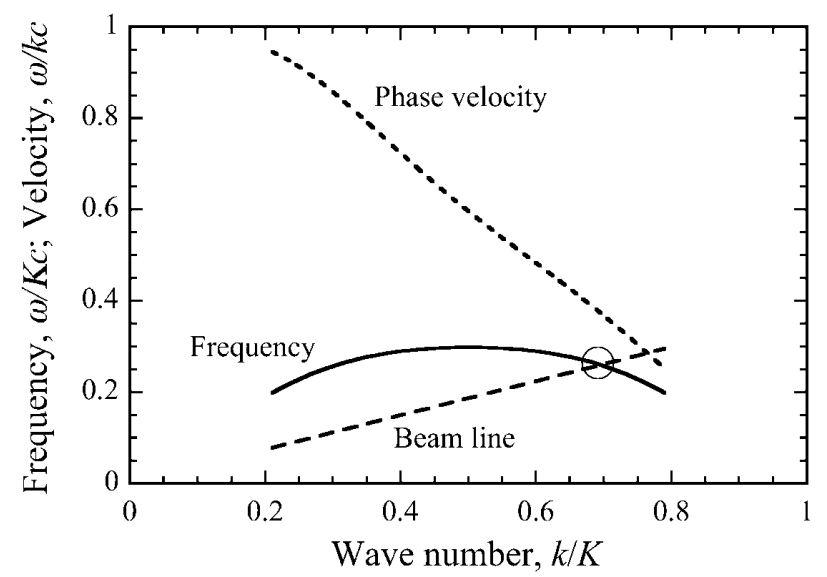

FIG. 2. Frequency (solid line) and phase velocity (dotted line) of the evanescent wave for the grating used by Urata and Walsh. The dashed line is the beam line $(\beta k / K)$ for $40 \mathrm{keV}$ electrons. The operating point for $40 \mathrm{keV}$ electrons (circled) is the intersection of the frequency curve with the beam line. in the matrix of coefficients $C_{m n}$, provided that at least three terms are used in the sum for the coefficients (that is, $-1 \leq p \leq 1$ ). It seems that the field in the grooves is adequately represented by a single term $(n=0)$, at least for $k<K$, but that the evanescent field must at least minimally reflect the periodicity of the grating.

To compute the gain, we take advantage of this simplification, and examine the dispersion relation

$$
R_{00}-1+\chi_{0} S_{00}=0 \text {. }
$$

When the effect of the electron beam is small, we expand about the solution for the no-beam case and write

$$
R_{00}(\omega, k) \approx R_{00}\left(\omega_{0}, k_{0}\right)+R_{00}^{\prime}\left(\omega_{0}, k_{0}\right)\left(k-k_{0}\right),
$$

where

$$
R_{00}\left(\omega_{0}, k_{0}\right)=1 .
$$

To lowest order, then, we are left with the equation

$$
R_{00}^{\prime}\left(\omega_{0}, k_{0}\right)\left(k-k_{0}\right)+\chi_{0} S_{00}\left(\omega_{0}, k_{0}\right)=0 .
$$

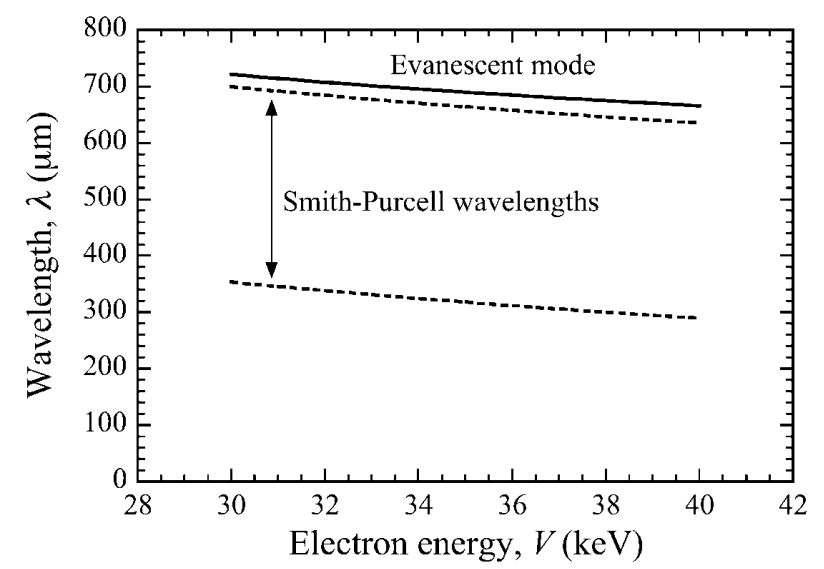

FIG. 3. Free-space wavelength of the evanescent mode (solid line), and angle-tuning wavelength range of Smith-Purcell radiation (region between the dotted lines), as functions of the electron-beam energy for the parameters of Urata and Walsh. 
TABLE I. Parameters of a Smith-Purcell FEL, from the data of Urata and Walsh.

\begin{tabular}{lc}
\hline \hline Grating period & $173 \mu \mathrm{m}$ \\
Groove width & $62 \mu \mathrm{m}$ \\
Groove depth & $100 \mu \mathrm{m}$ \\
Electron energy & $30-40 \mathrm{keV}$ \\
Electron-beam current & $1 \mathrm{~mA}$ \\
Electron-beam diameter & $24 \mu \mathrm{m}$ \\
\hline \hline
\end{tabular}

But the susceptibility diverges near the synchronous point, so the gain is largest there. We therefore select the point

$$
\omega_{0}=\beta c k_{0}
$$

and expand

$$
\chi_{0}=-\frac{\omega_{p}^{2}}{\gamma^{3}\left(\omega_{0}-\beta c k\right)^{2}}=\frac{\omega_{p}^{2}}{\gamma^{3} \beta^{2} c^{2}\left(k-k_{0}\right)^{2}} .
$$

Substituting this back into (37) we get

$$
\left(k-k_{0}\right)^{3}=\frac{\omega_{p}^{2}}{\gamma^{3} \beta^{2} c^{2}} \frac{S_{00}\left(\omega_{0}, k_{0}\right)}{R_{00}^{\prime}\left(\omega_{0}, k_{0}\right)} .
$$

Of the three roots, the root with the largest negative imaginary part has the highest gain, and we find that the amplitude growth rate is

$$
\mu=\operatorname{Im}\left(k-k_{0}\right)=\frac{\sqrt{3}}{2}\left|\frac{\omega_{p}^{2}}{\gamma^{3} \beta^{2} c^{2}} \frac{S_{00}\left(\omega_{0}, k_{0}\right)}{R_{00}^{\prime}\left(\omega_{0}, k_{0}\right)}\right|^{1 / 3} .
$$

The growth rate for the power is twice this. When we carry out the differentiations and cancel common factors from $S_{00}$ and $R_{00}^{\prime}$, we arrive at the formula

$$
\mu=\frac{\sqrt{3}}{2}\left|\frac{\omega_{p}^{2}}{\gamma^{3} \beta^{2} c^{2}} \frac{G\left(\omega_{0}, k_{0}\right)}{F^{\prime}\left(\omega_{0}, k_{0}\right)}\right|^{1 / 3}
$$

where

$$
G(\omega, k)=\frac{\cos (k A)-1}{\alpha_{0} L k^{2} A^{2}}
$$

and

$$
\begin{aligned}
& F^{\prime}(\omega, k)=-\sum_{p=-\infty}^{\infty}\left\{\frac{A \sin [(k+p K) A]}{\alpha_{p} L(k+p K)^{2} A^{2}}\right. \\
& +\frac{\cos [(k+p K) A]-1}{\alpha_{p} L(k+p K)^{2} A^{2}}\left[\frac{k+p K}{\alpha_{p}^{2}}\right. \\
& \left.\left.+\frac{2}{k+p K}\right]\right\} \text {. }
\end{aligned}
$$

The power gain per pass is then

$$
g=e^{2 \mu Z},
$$

where $Z$ is the overall length of the grating.

\section{CONCLUSIONS}

To apply the present two-dimensional, uniform-beam model to the real-world situation of Urata and Walsh, we assume that the electron beam uniformly fills a region of diameter $d_{e}$, so the plasma frequency in the beam is

$$
\omega_{p}^{2}=\frac{16 c^{2}}{\beta d_{e}^{2}} \frac{I_{e}}{I_{A}}
$$

where $I_{A}=4 \pi \varepsilon_{0} m c^{3} / q$ is the Alfvén current. We further assume that the uniform region of the electron beam is larger than the scale height, $s=1 / \alpha_{0}=\beta \gamma \lambda / 2 \pi$, of the evanescent wave and the width of the optical mode. The first assumption is probably satisfactory, but the width of the optical mode may be much larger than the electronbeam diameter, especially in low-gain cases [24]. With these assumptions we find that the amplitude growth rate is given by the formula

$$
\mu=\frac{\sqrt{3}}{\beta \gamma}\left|\frac{4 \pi}{d_{e}^{2} L} \frac{I_{e}}{I_{A}} \frac{G\left(\omega_{0}, k_{0}\right)}{F^{\prime}\left(\omega_{0}, k_{0}\right)}\right|^{1 / 3}
$$

Some computations using these formulas for the experimental parameters used by Urata and Walsh (Table I) are shown in Figs. 4 and 5. Urata and Walsh did not measure the gain directly, but if it is supposed that the traveling wave reflects off the ends of the grating in the manner of an optical resonator with high (say 90\%) output coupling at each end, then the gain per pass at threshold might be on the order of 100. This agrees roughly with the present computations. More recently, Bakhtyari, Walsh, and Brownell have examined in detail the transition from linear to nonlinear behavior of the radiation near threshold [25]. They observe oscillations in the radiated power near threshold, which they interpret as beating between three modes corresponding to the three

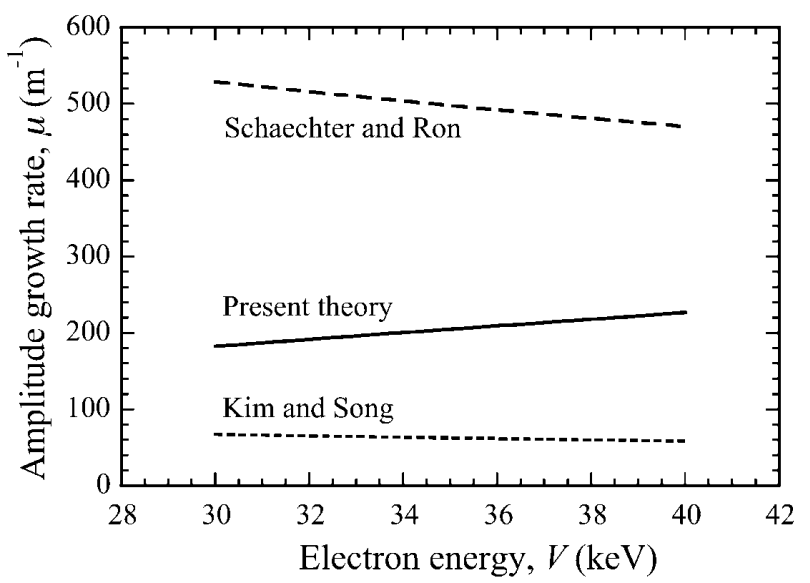

FIG. 4. Amplitude growth rate (per unit distance) for the experimental parameters of Urata and Walsh: present theory (solid line), Kim and Song (dotted line), Schaechter and Ron (dashed line). 


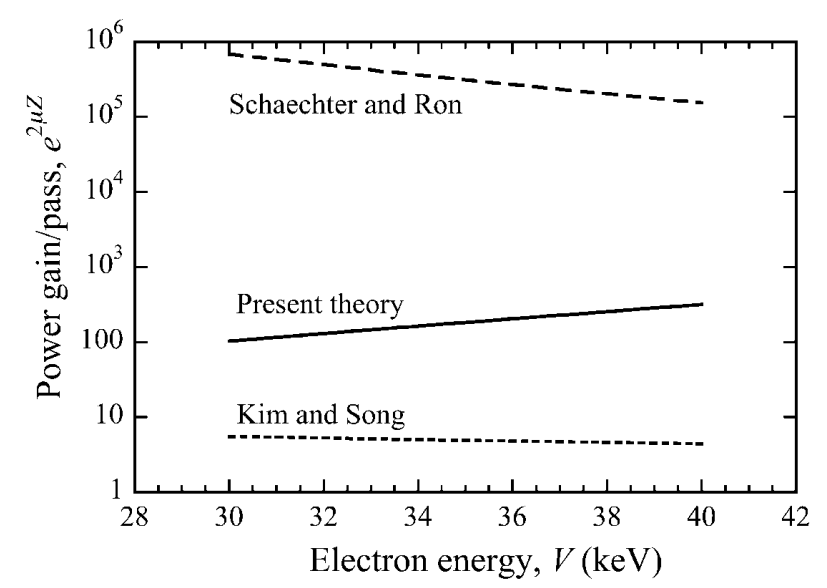

FIG. 5. Total power gain per pass for the experimental parameters of Urata and Walsh: present theory (solid line), Kim and Song (dotted line), Schaechter and Ron (dashed line).

roots of the dispersion relation. From these beats, they infer that the gain is proportional to the cube root of the current, as found in the present analysis.

Two other theories have been proposed to compute the gain of a Smith-Purcell FEL. Schaechter and Ron analyze the interaction of an electron beam with a wave traveling along the grating, and include waves that are emitted by the beam and reflected off the grating [19]. They treat the system as an amplifier, calculate the rate of growth of a wave that is incident on the grating from infinity, and arrive at the formula

$$
\mu=\frac{\sqrt{3}}{2}\left|\frac{4 \pi}{d_{e}} \frac{\omega^{2}}{c^{2}} \frac{e^{-2 \alpha_{0} h}}{\gamma^{5} \beta^{5}} \frac{I_{e}}{I_{A}}\right|^{1 / 3},
$$

where $h$ is the height of the beam above the surface of the grating. Compared with (47), the different dependence of the growth rate on the diameter of the electron beam follows from the fact that the beam is assumed by Schaechter and Ron to be a sheet of width $d_{e}$ positioned above the grating at the height $h$. For a finite-sized beam traveling as close to the grating as possible, we take $h=$ $d_{e} / 2$. The results of computations using this formula are shown for comparison in Figs. 4 and 5. Another theory has been advanced by Kim and Song [20]. Like Schaechter and Ron, they consider a sheet electron beam of width $d_{e}$ positioned at a height $h$ above the grating that interacts with a Floquet wave traveling along the surface of the grating, somewhat like the present theory. However, they assume that at least one of the Fourier components of the Floquet wave is radiative, rather than evanescent. That is, at least one component of the wave is not exponentially decreasing away from the grating surface. For the gain coefficient they obtain the formula

$$
\mu=\frac{1}{\gamma^{2} \beta^{2}} \sqrt{\frac{2 \pi k e_{00} e^{-2 \alpha_{0} h}}{d_{e}} \frac{I_{e}}{I_{A}}},
$$

where $e_{00}$ is a grating coupling coefficient whose value is of the order of unity. The results of computations using this formula are also shown for comparison in Figs. 4 and 5. The predicted gain is lower than that of the other theories, and depends on the square root of the electron-beam current rather than the cube root, as predicted by the other theories and inferred by Bakhtyari, Walsh, and Brownell. The different dependence of the gain on the electron-beam current may be due to the fact that Kim and Song assume that at least one component of the Floquet wave radiates as it travels along the grating, and this introduces a loss mechanism that is not present in the other theories.

It is interesting to note that the present theory predicts that the gain increases with electron energy, whereas the theories of Schaechter and Ron and of Kim and Song predict that the gain decreases. In the present theory, the gain increases with electron energy due to the dispersion relation, which is explicitly included in the present theory but not in the others. In a fundamental sense, the net gain for the evanescent wave is a balance between the energy absorbed from the electron beam and that lost by energy flow along the grating. But the energy in the evanescent wave travels at the group velocity, $d \omega / d k$, and this depends on the wave number of the wave, as indicated in Fig. 2. For the conditions examined by Urata and Walsh, the group velocity is actually negative $(d \omega / d k<0)$, as mentioned earlier. As the electron energy increases over the range explored by Urata and Walsh, the absolute magnitude of the group velocity decreases, so the gain increases.

The model used here suggests some interesting conclusions about the fundamental mechanism of a SmithPurcell FEL and the radiation that has been observed in the experiments of Urata and Walsh. Computations show that the laser frequency, which corresponds physically to the bunching frequency of the electron beam, is below the frequency range of Smith-Purcell radiation at any angle, as indicated in Fig. 3. Thus, the superradiant emission observed by Urata and Walsh in the direction normal to the grating is actually Smith-Purcell radiation enhanced by the nonlinear bunching of the electron beam induced by the interaction with the evanescent wave. This might be confirmed experimentally either by observing the variation of the frequency of the radiation with the angle of observation $\theta$ (there can be only one laser frequency, which is the frequency of the electron bunching), or by looking for radiation at the bunching frequency due to outcoupling of the evanescent wave at the ends of the grating. For example, in the experiments of Urata and Walsh, the Smith-Purcell radiation emitted normal to the grating by a $35 \mathrm{keV}$ electron beam is observed at $\lambda=$ $490 \mu \mathrm{m}$, whereas the evanescent wave is predicted to appear at the wavelength $2 \pi c / \omega=690 \mu \mathrm{m}$.

These results suggest that a Smith-Purcell FEL might be designed to optimize either the Smith-Purcell 
radiation or the evanescent wave. If the ends of the grating are constructed to reflect the evanescent mode, as might be done by tapering the grating period to form Bragg reflectors, then the evanescent mode will grow to saturation and the Smith-Purcell radiation will be enhanced by strong bunching. This would offer the advantages of angle tuning, in addition to tuning by the electron energy, and even multiple simultaneous wavelengths. However, the radiation at any given frequency would be reduced by the distribution of the Smith-Purcell radiation over a range of wavelengths. Alternatively, one or both ends of the grating could be used to outcouple the energy in the evanescent mode, making the Smith-Purcell FEL operate in the manner of a backward-wave oscillator. This would have the advantage of putting all the energy in a single wavelength, but the outcoupled energy would appear at a longer wavelength.

\section{ACKNOWLEDGMENTS}

The authors gratefully acknowledge helpful discussions with Hayden Brownell and Charles Boulware. This work was supported by the Medical Free Electron Laser Program of the Department of Defense under Grant No. F49620-01-1-0429.

[1] S. P. Mickan and X.-C. Zhang, Int. J. High Speed Electron. 13, 601 (2003).

[2] E. Brundermann, D. R. Chamberin, and E. E. Haller., Infrared Phys. Technol. 40, 141 (1999).

[3] R. Koehler, A. Tredicucci, F. Beltram, H. E. Beere, E. H. Linfield, A. G. Davies, D. A. Ritchie, R. C. Iotti, and F. Rossi, Nature (London) 417, 156 (2002).

[4] A. Bonvalet and M. Joffre, in Femtosecond Laser Pulses, edited by C. Rulliere (Springer-Verlag, Berlin, 1998).
[5] X.-C. Zhang and D. H. Auston, J. Appl. Phys. 71, 326 (1992).

[6] Ch. Fattinger and D. Grischkowsky, Appl. Phys. Lett. 53, 1480 (1988).

[7] Kenneth J. Button, Infrared and Millimeter Waves (Academic, New York, 1979).

[8] M. Abo-Bakr, J. Feikes, K. Holldack, G. Wuestefeld, and H.-W. Huebers, Phys. Rev. Lett. 88, 254801 (2002).

[9] G. P. Williams, Rev. Sci. Instrum. 73, 1461 (2002).

[10] G. Ramian, Nucl. Instrum. Methods Phys. Res., Sect. A 318, 225 (1992).

[11] A. Doria, G. P. Gallerano, E. Giovenale, G. Messina, and I. Spassovsky (to be published).

[12] J. Urata, M. Goldstein, M. F. Kimmitt, A. Naumov, C. Platt, and J. E. Walsh, Phys. Rev. Lett. 80, 516 (1998).

[13] C. Hernandez Garcia and C. A. Brau, Nucl. Instrum. Methods Phys. Res., Sect. A 475, 559 (2001).

[14] P. M. van den Berg, J. Opt. Soc. Am. 63, 689 (1973).

[15] P. M. van den Berg, J. Opt. Soc. Am. 63, 1588 (1973).

[16] P. M. van den Berg and T. H. Tan, J. Opt. Soc. Am. 64, 325 (1974).

[17] J. R. Pierce, Traveling-Wave Tubes (Van Nostrand, New York, 1950).

[18] A. Gover and P. Sprangle, IEEE J. Quantum Electron. 17, 1196 (1981).

[19] L. Schaechter and A. Ron, Phys. Rev. A 40, 876 (1989).

[20] K.-J. Kim and S.-B. Song, Nucl. Instrum. Methods Phys. Res., Sect. A 475, 158 (2001).

[21] E. E. Fisch and J. E. Walsh, Appl. Phys. Lett. 60, 1298 (1992).

[22] C. A. Brau, Modern Problems in Classical Electrodynamics (Oxford University, New York, 2004), p. 342.

[23] C. A. Brau, Modern Problems in Classical Electrodynamics (Ref. [22]), pp. 291-292.

[24] C. A. Brau, Nucl. Instrum. Methods Phys. Res., Sect. A 407, 1 (1998).

[25] A. Bakhtyari, J. E. Walsh, and J. H. Brownell, Phys. Rev. E 65, 066503 (2002). 